\title{
References.
}

1. Lin JJ, et al. Ann Neurol. 2014 Aug 1. [Epub ahead of print].

\section{NEUROMETABOLIC CAUSES OF INFANTILE SPASMS}

Investigators at King Abdulaziz Medical City, Riyadh, Saudi Arabia, studied the prevalence of hereditary neurometabolic causes of infantile spasms in 80 cases presenting over a 15-year period. Of 10 patients (12.5\%) diagnosed with metabolic causes, 2 had a Leigh-like disorder, and 1 patient had each of the following diagnoses: ethylmalonic aciduria, nonketotic hyperglycinemia, hyperinsulinemic hypoglycemia, leukodystrophy, short-chain acyl-coenzyme A dehydrogenase deficiency, molybdenum cofactor deficiency, primary carnitine deficiency, and neonatal hypoglycemia due to panhypopituitarism. Most of the patients were born of consanguineous parents, and the hereditary group had a strong history of other family members affected. The typical hypsarrhythmia pattern in the EEG was more common in the hereditary metabolic group $(\mathrm{P}=0.003)$, and this group had a poor response to therapy $(\mathrm{P}=0.04)$. Metabolic disorders are a relatively common cause of infantile spasms in this subpopulation of Saudi patients. Early diagnosis with metabolic and genetic testing is important in selection of specific treatments and facilitating family counseling. (Alrifai MT, AlShaya MA, Abdulaban A, Alfadhel M. Hereditary neurometabolic causes of infantile spasms in 80 children presenting to a tertiary care center. Pediatr Neurol 2014 Sep;51(3):390-7).

COMMENTARY. In patients suspected of having a hereditary metabolic cause for infantile spasms, the authors recommend a more liberal application of advanced diagnostic techniques, such as whole exome sequencing, muscle biopsy for mitochondria biochemical and genetic studies, and newer neuroimaging techniques such as 3 Tesla MRI and PET scanning [1]. More extensive genetic testing is justified in higher risk populations where high consanguinity rates are prevalent. A review of etiology of infantile spasms in the United Kingdom where consanguinity is rare finds the common causes are hypoxic-ischemic encephalopathy (10\%), chromosomal anomalies (8\%), malformation $(8 \%)$, perinatal stroke (8\%), and tuberous sclerosis complex (7\%) [2][3].

\section{References.}

1. Alrifai MT, et al. Pediatr Neurol. 2014 Sep;51(3):390-7.

2. Pavone P, et al. Brain Dev. 2014 Oct;36(9):739-751.

3. Osborne JP, et al. Epilepsia. 2010 Oct;51(10):2168-74.

\section{DEVELOPMENTAL DISORDERS}

\section{AAP GENETICS DIAGNOSTIC APPROACH TO INTELLECTUAL DISABILITY OR GLOBAL DEVELOPMENTAL DELAY}

The American Academy of Pediatrics Committee on Genetics present a recommended clinical genetics diagnostic approach to the evaluation of intellectual disability or global developmental delays. The report addresses the advances in diagnosis and treatment of children with intellectual disabilities since the original AAP report in 
2006 [1]. Intellectual disability (ID) is characterized by significant limitations both in intellectual functioning and in adaptive behavior as expressed in conceptual, social and practical adaptive skills. The disability originates before age 18 years, and the prevalence is estimated at $1 \%$ to $3 \%$. Global development delay (GDD) is defined as a significant delay in 2 or more developmental domains, including gross or fine motor, speech/language, cognitive, social/personal, and activities of daily living, and is thought to predict a future diagnosis of ID. GDD is applied to younger children $<5$ years, and the prevalence is $1 \%$ to $3 \%$, similar to that of ID. The term ID is applied to older children for whom IQ testing is valid and reliable.

In patients with GDD or ID, chromosome microarray and fragile $\mathrm{X}$ are first line diagnostic tests. MRI remains important in some patients. The use of whole-exome sequencing as a diagnostic test is gaining popularity. (Moeschler JB, Shevell M. Committee on Genetics. Comprehensive evaluation of the child with intellectual disability or global development delays. Pediatrics 2014 Sep;134(3):e903-18).

COMMENTARY. The benefits of a comprehensive evaluation were studied in 20 families of children with ID, with and without an etiologic diagnosis [2]. A diagnosis was thought to help guide expectations and management and provide hope for treatment. Diagnosis assisted families in obtaining desired services, especially in schools. Families differed in their "need to know" a specific diagnosis, ranging from strong to indifferent. Families varied in their emotions and actions regarding prenatal genetic diagnosis. The importance of the pediatrician and clinical geneticist discussion is stressed before deciding on the best approach to the diagnostic evaluation. The pediatric neurologist has an equally important role in the arrival at the correct etiological diagnosis and management of a child with GDD or ID.

\section{References.}

1. Moeschler JB, Shdevell M. Pediatrics. 2006 Jun;117(6):2304-16.

2. Makela M, et al. Am J Med Genet A. 2009 Nov;149A(11):2393-402.

\section{SUBDURAL HEMORRHAGE AND ANTITHROMBOTIC THERAPY}

Investigators at the Mott Children's Hospital, Ann Arbor, MI, report 4 infants with diffuse brain injury and cerebral atrophy who developed cerebral venous sinus thrombosis and were treated with the low-molecular-weight heparin, enoxaparin. The infants subsequently developed subdural hemorrhages, diagnosed on routine MRI, and one had focal seizures. The use of enoxaparin in infants with diffuse brain injury has a risk of intracranial subdural hemorrhage. (Dang LT, Shavit JA, Singh RK, et al. Subdural hemorrhages associated with antithrombotic therapy in infants with cerebral atrophy. Pediatrics 2014 Sep;134(3):e889-93).

COMMENTARY. Asymptomatic subdural hematomas are common in infants with congenital heart disease and they resolve within 3 months of birth [1].

References.

1. Kelly P, et al. Pediatrics. 2014 Sep;134(3):e773-81. 